\title{
Electromagnetic Effects in the Near-Field Plume Exhaust of a Micro-Pulsed-Plasma Thruster
}

\author{
Michael Keidar* and Iain D. Boyd ${ }^{\dagger}$ \\ University of Michigan, Ann Arbor, Michigan 48109 \\ Erik Antonsen \\ University of Illinois at Urbana-Champaign, Urbana, Illinois 61801 \\ and \\ Gregory G. Spanjers ${ }^{\S}$ \\ Air Force Research Laboratory, Edwards Air Force Base, California 93524
}

\begin{abstract}
A model is presented of the near-field plasma-plume of a pulsed plasma thruster (PPT). As a working example, a micro-PPT developed at the U.S. Air Force Research Laboratory is considered. This is a miniaturized design of the axisymmetric PPT with a thrust in the $10-\mu \mathrm{N}$ range that utilizes Teflon ${ }^{\mathrm{TM}}$ as a propellant. The plasma plume is simulated using a hybrid fluid-particle-in-cell direct simulation Monte Carlo approach. The plasma plume model is combined with Teflon ablation and plasma generation models that provide boundary conditions for the plume. This approach provides a consistent description of the plasma flow from the surface into the near plume. The magnetic field diffusion into the plume region is also considered, and plasma acceleration by the electromagnetic mechanism is studied. Teflon ablation and plasma generation analyses show that the Teflon surface temperature and plasma parameters are strongly nonuniform in the radial direction. The plasma density near the propellant surface peaks at about $10^{24} \mathrm{~m}^{-3}$, whereas the electron temperature peaks at about $4 \mathrm{eV}$ near the electrodes. The plume simulation shows that a region with high density is developed at a few millimeters from the thruster exit plane at the axis. This high-density region exists during the entire pulse, but the plasma density decreases from about $2 \times 10^{22} \mathrm{~m}^{-3}$ at the beginning of the pulse down to $0.3 \times 10^{22} \mathrm{~m}^{-3}$ at $5 \mu \mathrm{s}$. The velocity phase is centered at about $20 \mathrm{~km} / \mathrm{s}$ in the axial direction. At later stages of the pulse, there are two ion populations with positive and negative radial velocity. Electron and neutral densities predicted by the plume model are compared with near-field measurements using a two-color interferometer, and good agreement is obtained.
\end{abstract}

\section{Nomenclature}

$\boldsymbol{B} \quad=$ magnetic field

$C_{s} \quad=$ sound speed

$E \quad=$ electric field

$j \quad=$ current density

$m \quad=$ heavy particle mass

$m_{e} \quad=$ electron mass

$N_{e} \quad=$ electron density

$N_{1}, N_{2}=$ densities

$Q_{F}=$ heat due to particle convection

$Q_{j} \quad=$ Ohmic heat

$Q_{r} \quad=$ radiation heat

$r_{a}=$ outer radius of the thruster tube

$T_{e} \quad=$ electron temperature, $\mathrm{eV}$

$T_{i} \quad=$ ion temperature, $\mathrm{eV}$

$T_{1}, T_{2}=$ temperatures

$V_{e} \quad=$ electron velocity

$V_{1} \quad=$ velocity at the Knudsen layer edge

$v_{\mathrm{ei}}=$ electron-ion collision frequency

$\Gamma \quad=$ ablation rate, $\mathrm{kg} / \mathrm{m}^{2} \cdot \mathrm{s}$

$\mu \quad=$ permittivity

Received 18 June 2002; accepted for publication 18 February 2004. Copyright (C) 2004 by the American Institute of Aeronautics and Astronautics, Inc. All rights reserved. Copies of this paper may be made for personal or internal use, on condition that the copier pay the $\$ 10.00$ per-copy fee to the Copyright Clearance Center, Inc., 222 Rosewood Drive, Danvers, MA 01923; include the code 0748-4658/04 \$10.00 in correspondence with the CCC.

${ }^{*}$ Senior Research Fellow, Department of Aerospace Engineering. Senior Member AIAA.

${ }^{\dagger}$ Professor, Department of Aerospace Engineering.

¥ Graduate Student, Department of Aerospace and Aeronautics. Student Member AIAA.

${ }^{\S}$ Scientist, Propulsion Directorate, Electric Propulsion Laboratory. $\sigma \quad=$ plasma (Spitzer) conductivity

$\omega \tau=$ Hall parameter

\section{Introduction}

$\mathbf{T}$ HE pulsed plasma thruster (PPT) was among the first of various electrical propulsion concepts accepted for space flight mainly due to its simplicity and, hence, high reliability. ${ }^{1}$ However, the PPT has an efficiency that is generally low $^{2}$ at about $10 \%$ leaving open the opportunity for considerable improvement. ${ }^{3}$ Currently, PPTs are considered as an attractive propulsion option for stationkeeping and drag makeup purposes of mass and power-limited satellites. ${ }^{4,5}$ Guaranteeing successful operation of spacecraft using a PPT requires a complete assessment of the spacecraft integration effects. The PPT plume contains various ion and neutral species due to propellant decomposition and possible electrode erosion. Some attempts of PPT plume modeling using particle simulations were performed recently. ${ }^{6-8}$ In Refs. 7 and 8 we have considered the plume flowfield exhaust from the recently developed electrothermal PPT (PPT-4), and therefore, electromagnetic effects in the plume were neglected. Different variations of electromagnetic PPTs are also candidates for various missions. ${ }^{9}$ For instance, an electromagnetic PPT was successfully operated for pitch axis control on the EO-1 spacecraft. ${ }^{10,11}$ Recently, an axisymmetric micro-PPT has been designed at the U.S. Air Force Research Laboratory (AFRL) for delivery of very small impulse bit. ${ }^{12}$ This is a simplified miniaturized version of a conventional PPT designed to provide attitude control and stationkeeping for microsatellites.

We will use the AFRL micro-PPT as a working example for several reasons. First, electromagnetic $(j \times B)$ acceleration is the primary mechanism in this thruster; second, there is no internal flow in this device, and therefore, the near-field plasma plume is an essential part of the thrust-generation process. Therefore, careful modeling of the acceleration is needed to understand the characteristics 


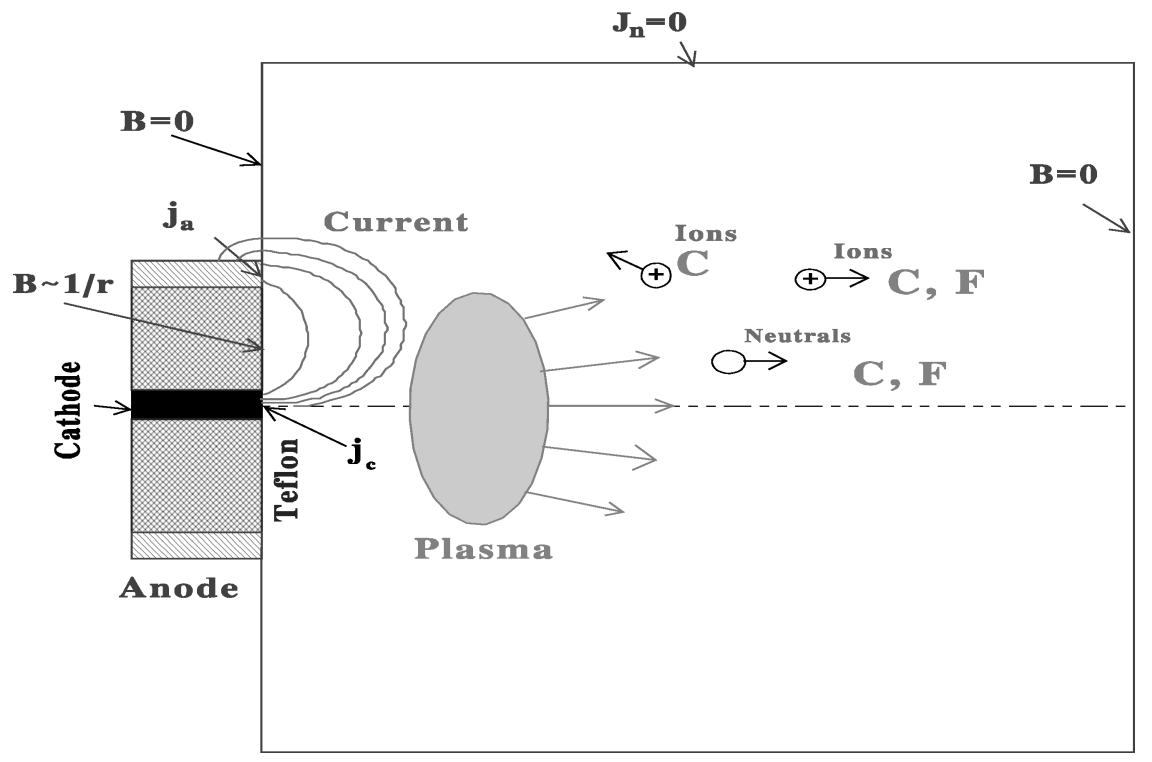

Fig. 1 Schematic of micro-PPT plume and boundary conditions.

of the device as a whole in addition to being a precursor to accurate estimation of contamination issues. Because in this device there is no separation between the main plasma acceleration region and the plume expansion, both regions must be simulated in one model. Because the plasma acceleration is external, the plasma is sufficiently rarefied so that a magnetohydrodynamic code such as MACH2 (Ref. 13) cannot be used in end-to-end simulation.

An accurate model of the PPT plume relies on the boundary and initial conditions. These conditions can be formulated by consideration of the Teflon ${ }^{\mathrm{TM}}$, ablation process. The Teflon ablation computation is based on a recently developed kinetic ablation model. ${ }^{14,15}$ In this model, we study details of the Teflon evaporation physics by consideration of the distribution function of the particles in the kinetic layer adjacent to the surface.

Another important effect related to the plasma plume exhaust from an electromagnetic PPT is the magnetic field diffusion into the near plume. Previously, we have modeled the effect of the magnetic field on the near-field plume for Hall thrusters under steady-state conditions (see Ref. 16). It was found that the magnitude of the magnetic field at the thruster exit has an important effect on the plasma potential distribution in the plume. In the present research, we include the electromagnetic effects on the near-field plume of unsteady plasma flow. The computational domain is shown in Fig. 1. The model is based on a hybrid approach involving a direct simulation Monte Carlo (DSMC) description of neutrals, a particle-in-cell (PIC) model for ions, and a fluid description of the electrons. In these methods, the potential distribution is usually calculated by reducing the electron momentum equation to the Boltzmann relation in the absence of a magnetic field. In the plasma plume domain where the magnetic field exists, that is, the near-field plume region, it is necessary to include the magnetic field effects in the electron momentum equation.

The rest of the paper is organized as follows. In Secs. II-IV, the model of the plasma generation is described in the framework of the fluid approach. The solution of this problem constitutes the boundary condition for the plume that is evaluated in Sec. V. In Sec. VI the results of the plasma plume simulation using the particle approach are presented and compared with experimental data (Sec. VII).

\section{Model of the Plasma Layer}

The model presented here describes the plasma layer near the Teflon surface as shown in Fig. 2. During the discharge, the plasma density near the propellant face is large [on the order of $10^{23}-10^{24}$ $\mathrm{m}^{-3}$ (Refs. 17 and 18)] and therefore, a fluid approach can be used. The plasma-layer model includes the following features (similar

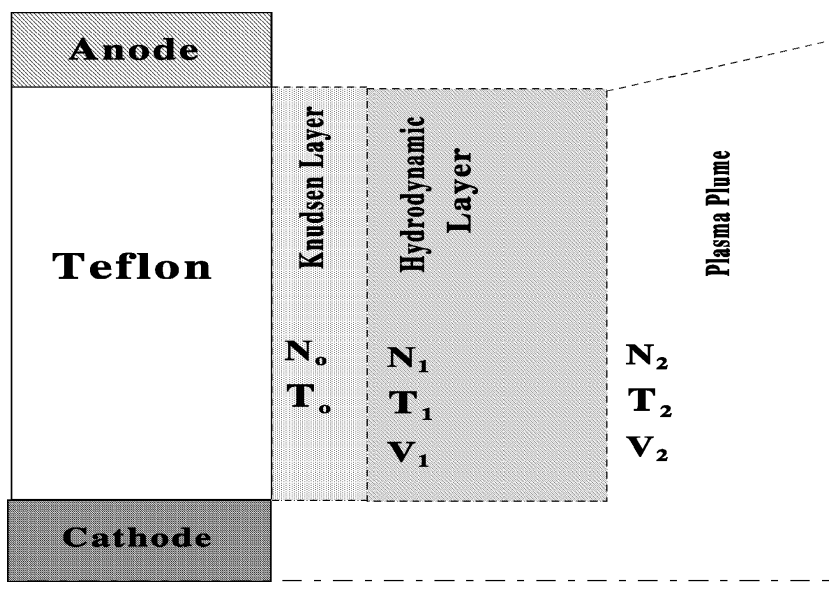

Fig. 2 Schematic of plasma layer.

to the model of an electrothermal $\mathrm{PPT}^{17,18}$ ): joule heating of the plasma, heat transfer to the Teflon surface, and Teflon ablation. Mechanisms of energy transfer from the plasma column to the wall of the Teflon include heat transfer by particle convection and by radiation. The Teflon ablation computation is based on a recently developed kinetic ablation mode ${ }^{14}$ (next section). It is assumed that within the plasma layer all parameters vary in the radial direction $r$. The electron energy balance equation can be written in the form ${ }^{17,18}$

$$
\frac{3}{2} N_{e} \frac{\mathrm{d} T_{e}}{\mathrm{~d} t}=Q_{J}-Q_{r}-Q_{F}
$$

A one-dimensional time-dependent model of the plasma layer is considered, and Eq. (1) depends on the coordinate $r$ along the propellant face (Fig. 1). The radiation heat flux $Q_{r}$ and particle convection heat flux $Q_{F}$ depend on the plasma density and temperature. ${ }^{17,18}$ According to Ref. 19, the radiation in the continuum from a $\mathrm{C}+2 \mathrm{~F}$ plasma in the considered parameter range provides the main contribution. The radiation energy flux $Q_{r}$ includes the radiation for a continuum spectrum based on a theoretical model. ${ }^{20,21}$ In the expression for $Q_{r}=A Z_{i}^{2} N_{e}^{2} T_{e}^{1 / 2}\left(1+\chi_{g}\right)$, the coefficient $A$ is a constant $\left(1.6 \times 10^{-38}\right.$ in SI units) and $\chi_{g}=E_{g} / T_{p}$ with $E_{g}$ as the energy of the lowest excited state. The particle convection flux $Q_{F}$ includes energy associated with electron and ion fluxes to the Teflon surface and out of the plume that leads to plasma cooling.

The Teflon surface temperature is calculated from the heat transfer equation with boundary conditions that take into account 
vaporization heat, surface radiation, and heat conductivity. Calculations show that surface radiation is negligibly small in the considered case. The solution of this equation is considered for two limiting cases of substantial and small ablation rate very similar to that described in Refs. 17 and 18. The density at the Teflon surface is calculated using the equilibrium pressure for Teflon. The plasma density in the layer is determined in the framework of the kinetic ablation model (next section). In many cases, for known pressure and electron temperature, one can calculate the chemical plasma composition assuming local thermodynamic equilibrium (LTE). $8,17,18,22,23$ Plasma parameters have also rapid temporal variation during the discharge pulse, so that there is a concern about the possibility of establishing LTE. For instance, in a homogeneous transient plasma, ${ }^{24}$ complete LTE may be obtained in $0.3 \mu$ s for a helium plasma with an electron density of $10^{24} \mathrm{~m}^{-3}$. An estimation of the characteristic times for ionization and recombination has shown that the ionization and recombination timescales for ground states of $\mathrm{C}$ and $\mathrm{F}$ are less than the typical time for discharge parameter changes. ${ }^{19}$ Therefore, LTE may be established during the discharge pulse (which is about few microseconds). An estimate of the relaxation time for elastic collisions has shown that, in plasma with a density of about $10^{22}-10^{24} \mathrm{~m}^{-3}$ and an electron temperature of $1-4 \mathrm{eV}$, equilibrium of electrons, ions, and neutrals is established on a timescale less than a microsecond (Ref. 25). For simplicity, only single ionization is considered within the LTE framework. However, it is shown in Sec. VII that, under significant plasma acceleration in the ionization layer, ionization equilibrium may not be established. Therefore, in addition, a more general approach of nonequilibrium ionization is considered. In the plasma layer, the ionization balance equation (Saha equation, or non-equilibrium ionization expression) is supplemented by the conservation of nuclei and quasi neutrality.

\section{Ablation Model}

The Teflon ablation is modeled in the framework of the approximation ${ }^{15}$ based on a kinetic model of the material evaporation into discharge plasmas. ${ }^{14}$ The model couples two different layers between the surface and the plasma bulk as shown in Fig. 2: 1) a kinetic nonequilibrium layer adjusted to the surface with a thickness of about one mean free path and 2) a collision-dominated layer with thermal and ionization nonequilibrium. The velocity at the edge of the kinetic layer $V_{1}$ can be determined from the coupling solution of the hydrodynamic layer and the quasi-neutral plasma. For known velocity and density at this interface, it is possible to calculate the ablation rate. In the hydrodynamic layer, the relation between the velocities, temperatures, and densities at boundaries 1 and 2 as well as the ablation rate are formulated according to Ref. 15 in the form

$$
\begin{aligned}
\Gamma= & m V_{1} N_{1}=N_{1}\left[\left(2 k T_{1} / m\right)\right. \\
& \left.\cdot\left(T_{2} N_{2} / 2 T_{1}-N_{1} / 2\right) /\left(N_{1}-N_{1}^{2} / N_{2}\right)\right]^{0.5}
\end{aligned}
$$

The system of equations is closed if the equilibrium vapor pressure can be specified that determines parameters $N_{0}$ and $T_{0}$ at the Teflon surface. The solution of the Knudsen layer problem relates parameters at boundary 1 to the parameters at boundary 0 (Ref. 14). The full self-consistent solution of this problem can be obtained when the ablation is coupled with the plasma plume expansion. In the present work, to simplify the problem, we will assume that the plasma accelerates up to the sound speed near boundary 2 . This assumption can be justified by that, due to significant electromagnetic acceleration in this type of PPT, the plasma density will quickly decrease, therefore, providing a solution of the ablation problem close to that for ablation into vacuum. In this case, the plasma density at the edge of the kinetic layer will be equal to $0.34 \cdot N_{0}$ and the temperature is $0.7 \cdot T_{0}$. The flux returned to the surface is equal to $16 \%$ of the ablated flux (Ref. 14).

\section{Plasma Plume Electrodynamics}

The general approach for the plume model is based on a hybrid fluid-particle approach that was used previously (Refs. 7 and 8). In this model, the neutrals and ions are modeled as particles, whereas electrons are treated as a fluid. Elastic (momentum transfer) and nonelastic (charge exchange) collisions are included in the model. The particle collisions are calculated using the DSMC method. ${ }^{26}$ Momentum exchange cross sections use the model of Dalgarno et al., ${ }^{27}$ whereas charge exchange processes use the cross sections proposed by Sakabe and Izawa. ${ }^{28}$ Acceleration of the charged particles is computed using the PIC method. ${ }^{29}$ A single grid employing nonuniform, rectangular cells is used for both the DSMC and PIC steps. Because the flow is assumed to be quasi neutral, there is no requirement to resolve the Debye length. Hence, the cells are scaled by the local mean free path. A single time step given by the reciprocal of the maximum plasma frequency is used throughout.

The electron dynamics is very important in the plasma plume. Previously our model was based on the assumption that electrons rapidly reach the equilibrium distribution and in the absence of the magnetic field can be described according to the Boltzmann distribution (see Ref. 7). Although this was a satisfactory assumption in the case of an electrothermal thruster plume, this is not suitable for the near field of an electromagnetic thruster. In the presence of a strong magnetic field, the electron density distribution deviates from that of the Boltzmann distribution (see Ref. 30). In the case of a magnetic field, the electron momentum equation is (neglecting electron inertia)

$$
0=-e N_{e}\left(\boldsymbol{E}+\boldsymbol{V}_{e} \times \boldsymbol{B}\right)-\nabla P_{e}-N_{e} v_{\mathrm{ei}} m_{e}\left(\boldsymbol{V}_{e}-\boldsymbol{V}_{i}\right)
$$

We have assumed quasineutrality; therefore, $N_{e}=N_{i}=N$. When a definition $\boldsymbol{j}=e N\left(\boldsymbol{V}_{e}-\boldsymbol{V}_{i}\right)$ is used, Eq. 3 is usually referred to as the generalized Ohm's law. The electric and magnetic field distributions in the plume can be calculated from the set of Maxwell's equations. We further assume that the magnetic field has only an azimuthal component and also neglect the displacement current. The combination of Maxwell's equations and electron momentum conservation gives the following equation for the magnetic field:

$$
\frac{\partial \boldsymbol{B}}{\partial t}=\frac{1}{(\sigma \mu)} \nabla^{2} \boldsymbol{B}-\nabla \times\left(\frac{\boldsymbol{j} \times \boldsymbol{B}}{(e N)}\right)+\nabla \times(\boldsymbol{V} \times \boldsymbol{B})
$$

where $\sigma=e^{2} N_{e} /\left(v_{\mathrm{ei}} m_{e}\right)$ is the plasma conductivity, which has only a weak dependence on the plasma density (in the Coulomb logarithm). Therefore, it was assumed that plasma conductivity depends only on the electron temperature in the plasma plume. A similar approach was also recently employed in the plasma flow simulation in the PPT. ${ }^{31}$ The electron temperature was calculated from the plasma layer fluid model. (See Secs. II and VI.) It was assumed in Eq. (4) that the plasma conductivity is constant (mainly because electron temperature is assumed to be constant in the near plume ${ }^{7}$ ) and that the density gradient does not affect the magnetic field diffusion. The last assumption comes from the fact that the main density gradient is developed in the direction of magnetic field transport (as clear from the later results) and, therefore, does not affect magnetic field transport. ${ }^{32}$

A scaling analysis shows that, in the case of the near plume of the micro-PPT with a characteristic scale length $L$ of about $1 \mathrm{~mm}$, the magnetic Reynolds number $\operatorname{Re}_{m}=\mu \sigma L V \ll 1$ (where $V$ is the characteristic velocity $\sim 10^{4} \mathrm{~m} / \mathrm{s}$ as shown later); therefore, the last term in Eq. (4) can be neglected. In addition, depending on the plasma density, the Hall effect [second term on the right-hand side of Eq. (4)] may be important for the magnetic field evolution. One of the first calculations of the plasma flow with Hall effect was performed by Brushlinski and Morozov, ${ }^{33}$ who considered isothermal flow. The plasma density becomes high at the cathode and lower at the anode. The Hall effect has a particularly noticeable influence on the magnetic field distribution. The field near the anode increases and near the cathode decreases. As a result, the current is deflected to the side and grazes the anode. Our estimations show that the Hall parameter $\omega \tau \ll 1$ if the plasma density near the Teflon surface $N>10^{23} \mathrm{~m}^{-3}$. This is usually the case in the micro-PPT (next section) so that the Hall effect is expected to be small and will not be considered in this paper. Therefore, Eq. (5) is reduced to the simple 
magnetic transport equation,

$$
\frac{\partial \boldsymbol{B}}{\partial t}=\frac{1}{(6 \mu)} \nabla^{2} \boldsymbol{B}
$$

Having the magnetic field distribution, one can calculate the current density distribution from Ampere's law:

$$
\mu \boldsymbol{j}=\nabla \times \boldsymbol{B}
$$

The magnetic field and current distributions calculated from this model are used in PIC to evaluate the ion dynamics. The ion velocity distribution depends on the magnetic field distribution, and the ion dynamics is calculated as follows:

$$
\frac{m \mathrm{~d} \boldsymbol{V}}{\mathrm{d} t}=Z_{i} e(\boldsymbol{E}+\boldsymbol{V} \times \boldsymbol{B})+v_{\mathrm{ei}} m_{e}\left(\boldsymbol{V}_{e}-\boldsymbol{V}_{i}\right)
$$

The electric field in this equation can be determined from the electron momentum equation (3), and therefore, the last equation reduces to the following simplified form:

$$
\frac{m \mathrm{~d} \boldsymbol{V}}{\mathrm{d} t}=\boldsymbol{j} \times \frac{\boldsymbol{B}}{N}
$$

\section{Boundary Conditions}

Here, we present an overall end-to-end model of the plasma generation and near-field plume expansion in the micro-PPT. This model considers separately two regions, namely, a plasma-generation region and a plasma plume expansion near-field region. The solution of the plasma-generation region provides a boundary condition for the plasma plume expansion.

The boundary conditions for the magnetic field calculations are shown in Fig. 1. We assume that the current is uniform on both electrodes, and that allows us to calculate the current density on the cathode $j_{c}$ and on the anode $j_{a}$. The magnetic field is assumed to vary as $1 / r$ between electrodes and $B=0$ outside of the electrodes. At the lateral boundary, we assume that the normal current $j_{n}=0$. The downstream boundary is considered to be far enough away that $B=0$ can be assumed. Along the centerline, the magnetic field is zero.

The boundary conditions for the plume are generated through solution of the Teflon ablation problem as will be presented in the Results section. These involve time and radial dependent variations of the plasma (including carbon and fluorine ions and neutrals) density and electron temperature.

\section{Results}

The results are presented for a 3.6-mm- (0.141-in.-) diam axisymmetric micro-PPT, which has a 0.9 -mm-diam central electrode, 3.1-mm propellant diameter, and 0.24-mm anode wall (Ref. 12). In these simulations, the experimental current waveform is used, and it can be described in a first approximation as an underdamped circuit current:

$$
I(t)=I_{p} \cdot \sin (\Omega t) \exp (-\gamma t)
$$

where $I_{p}=\sqrt{ }(2 E / L), \Omega=\sqrt{ }(1 / L C), \gamma=R / 2 L, L$ is the effective inductance in the circuit, $C$ is the capacitance, $R$ is the total circuit resistance, and $E$ is the pulse energy. Results to be presented correspond to $E=2.25 \mathrm{~J}$ and $C=0.5 \mu \mathrm{F}$. The best fit with the experimental waveform ${ }^{12}$ (frequency) corresponds to $\alpha=4.7 \times 10^{7} \mathrm{rad} / \mathrm{s}$ and circuit inductance $L=90 \mathrm{nH}$ and results in a peak current of $3.5 \mathrm{kA}$ at $t=0.4 \mu \mathrm{s}$.

The plasma density and electron temperature distribution are shown in Figs. 3a and 3b. The plasma density peaks at about $10^{24} \mathrm{~m}^{-3}$. The electron temperature is strongly nonuniform radially with peaks near the electrodes of about $4.5 \mathrm{eV}$ as shown in Fig. 3b. The reason for higher electron temperature near the electrodes is due to current spreading in the space between the electrodes and current focusing near the electrodes. The plasma pressure in the propellant vicinity can be calculated from the density and electron temperature distribution, and it has a peak at about $10 \mathrm{~atm}$.

The spatial and temporal variation of the Teflon surface temperature is shown in Fig. 3c. The Teflon temperature sharply increases during the first $2 \mu$ s of the pulse and peaks at about $990 \mathrm{~K}$. One can see that the temperature is generally nonuniform in the radial direction and has a minimum at radial distances of $1.1-1.3 \mathrm{~mm}$. Because the Teflon ablation is approximately exponentially proportional to the surface temperature, the model predicts a lower rate of ablation in the areas where the surface temperature has a minimum. When this is taken into account, the effect of the radially nonuniform temperature distribution may be related to the preferential charring of the Teflon surface observed experimentally. ${ }^{12}$ A detailed study of the Teflon surface charring and its relation to the nonuniform temperature distribution and ablation is presented in a parallel paper. ${ }^{34}$ As mentioned earlier, the ablation rate is also nonuniform radially. This effect is shown in Fig. 3d. The calculated total ablated mass per pulse is about $1.4 \mu \mathrm{g}$.

A region of magnetic field diffusion in the near field outside the micro-PPT is shown in Fig. 4. The magnetic field drops by an order of magnitude at about $1.5 \mathrm{~mm}$ downstream, a distance equal to the thruster radius. This is also the region where most of the current is concentrated.

Figure 5 shows evolution of the carbon ion $(\mathrm{C}+)$ component of the plasma plume during the main part of the pulse. One can see that a high-density region is developed a few millimeters from the thruster exit plane. This dense region exists during the entire pulse as shown in Fig. 5, but the plasma density decreases from about $2 \times 10^{22} \mathrm{~m}^{-3}$ at the beginning of the pulse down to $0.3 \times 10^{22} \mathrm{~m}^{-3}$ at $5 \mu \mathrm{s}$. At the beginning (first $2 \mu \mathrm{s}$ ), the $\mathrm{C}+$ density mainly develops a gradient in the radial direction that is a result of high directed velocity in the axial direction. Later, during the pulse, the axial density gradient becomes comparable to the radial one.

The fluorine ions $(\mathrm{F}+)$, due to their larger mass, have different dynamics, as shown in Fig. 6. They have smaller acceleration in the axial direction even at the beginning of the pulse, and therefore, both axial and radial density gradients are developed. The F+ density in the plume and in the high-plasma-density region is larger than that of $\mathrm{C}+$ because originally Teflon has composition $\mathrm{C}_{2} \mathrm{~F}_{4}$ with $\mathrm{F}$ density twice that of $\mathrm{C}$. Additionally $\mathrm{F}$ ions experience less acceleration in the plume because of their higher mass, which also contributes to their relative density increase.

The micro-PPT is essentially an electromagnetic accelerator, as shown in the velocity phase plots (Figs. 7 and 8). The phase plot of the carbon ions at $1 \mu \mathrm{s}$ is centered at $20 \mathrm{~km} / \mathrm{s}$ in the axial direction. (Note that the sound speed is about $5 \mathrm{~km} / \mathrm{s}$ for a $4-\mathrm{eV}$ plasma.) Ions also experience radial expansion in both directions due to the magnetic field structure and the temperature expansion. The radial velocity in the negative direction is related to the focus formation along the axis, as shown in Figs. 5 and 6. The fluorine ions have generally smaller both axial and radial velocities due to their higher mass. At a later stage of the pulse (Fig. 8), clearly there are two ion populations with positive and negative radial velocities. This is due to the annular plasma injection corresponding to the thruster geometry (Figs. 1 and 2).

During the entire pulse there is a population of ions having a negative axial velocity with magnitude up to about $10 \mathrm{~km} / \mathrm{s}$ (Figs. 7 and 8). This population creates the backflow contamination that occurs mainly onto the thruster itself. The carbon ions have a larger negative velocity due to their higher mobility, which results in their domination in the backflux. This backflux may be mainly responsible for charring phenomena observed in this thruster. ${ }^{12,34}$

\section{Comparison with Experiment}

In this section, we present measured and predicted electron and neutral density distributions in the near-field plume for one microPPT design. These data will be compared to assess our plume and device model. It is expected that nonequilibrium ionization effects 


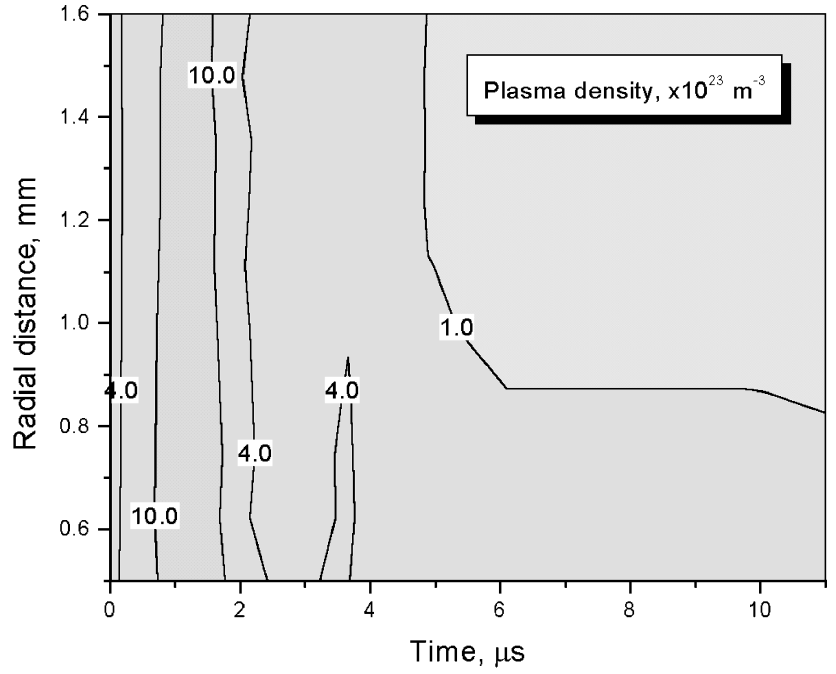

a)

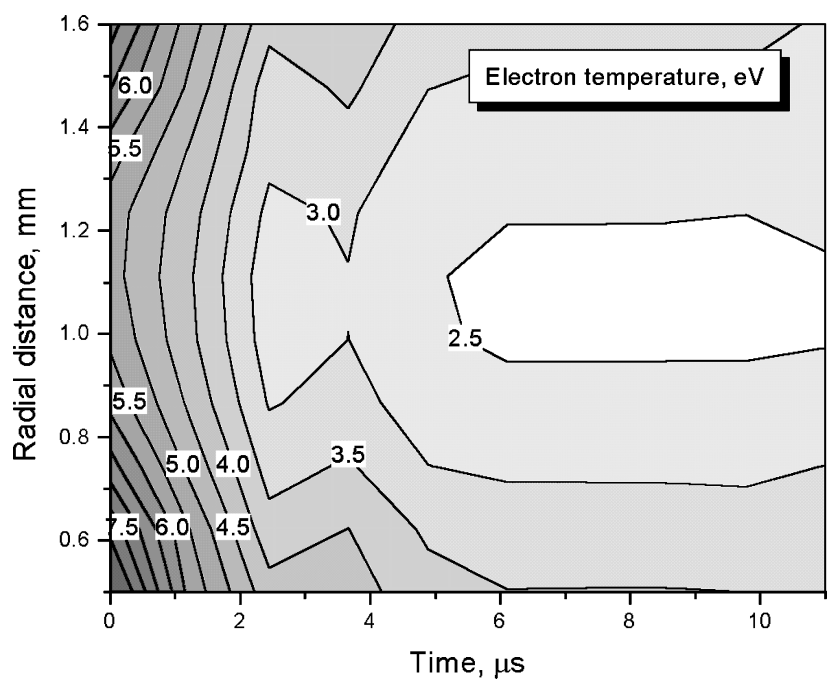

b)

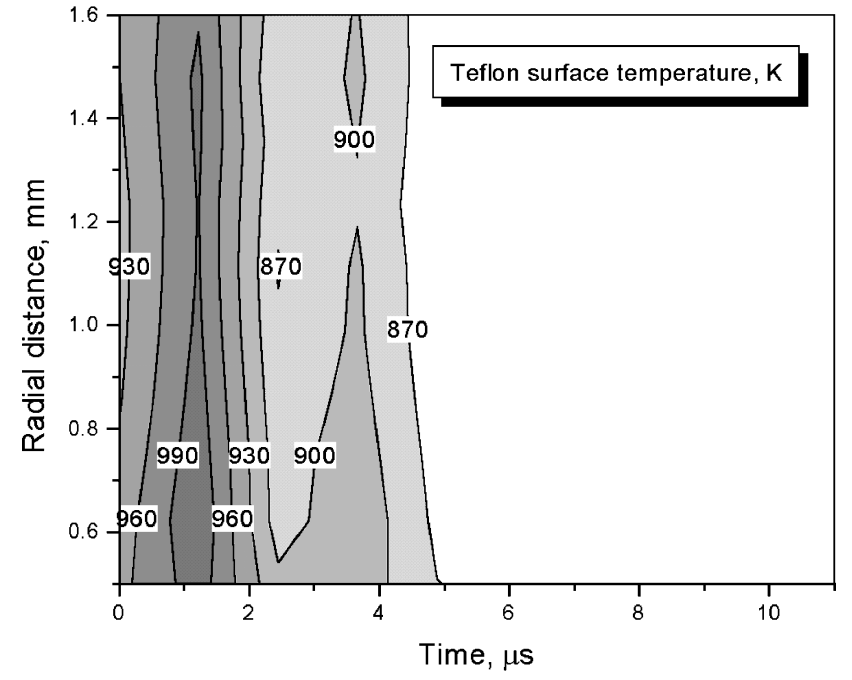

c)

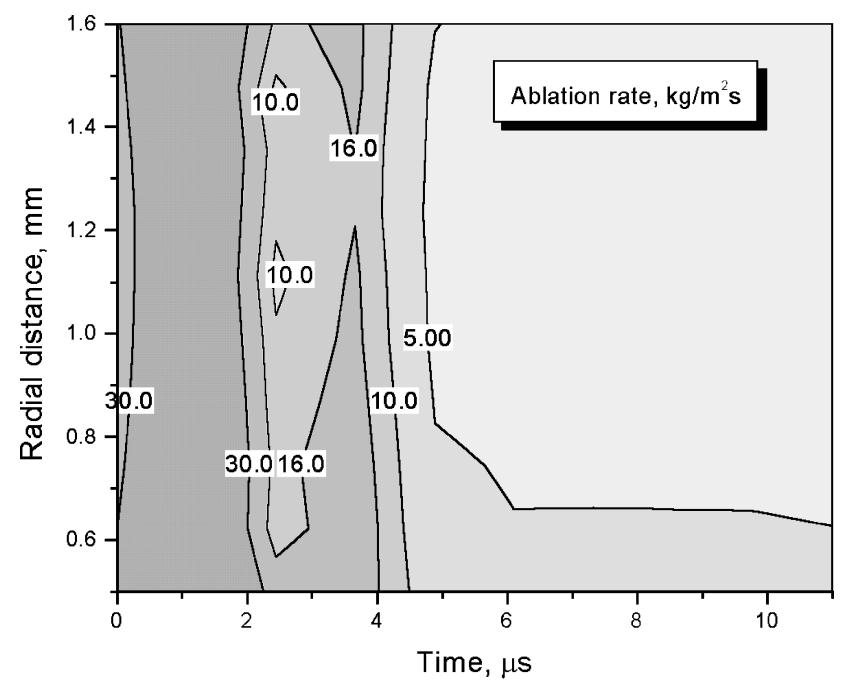

d)

Fig. 3 Spatiotemporal distribution: a) Teflon surface temperature, b) plasma density, c) electron temperature, and d) ablation rate.

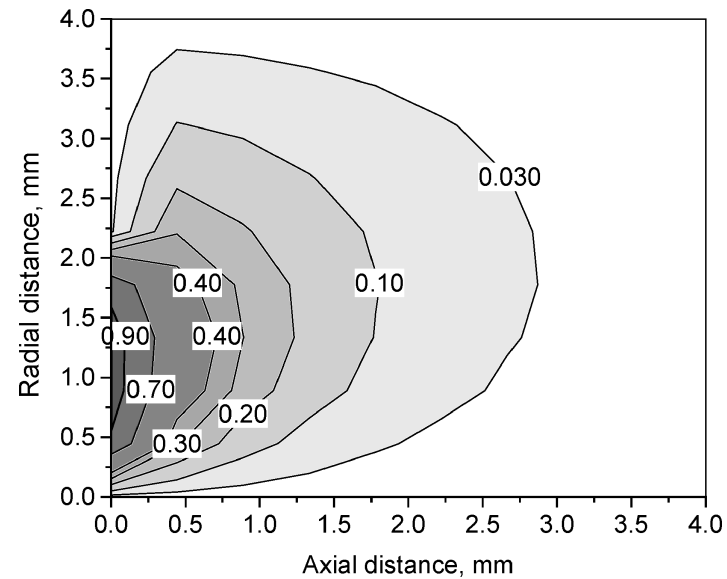

Fig. 4 Magnetic field distribution in the near field of the micro-PPT.

play an important role due to electromagnetic acceleration taking place in the plasma-generation region. Whereas general features of the plasma plume remain very similar to ones presented earlier (based on the LTE plasma-generation model), the ionization degree is strongly affected by an ionization model. Therefore, for the purpose of comparison with experimental data, we use a more general plasma-generation model that is based on nonequilibrium ionization.

\section{A. Nonequilibrium Ionization Model}

The ionization layer can be physically determined as follows. At the beginning of the ionization layer, the charged particle density is very small, and therefore, ionization becomes the leading process because recombination has higher order dependence on the electron density. As charged particle density grows, the recombination rate increases, and near the edge of the layer the recombination rate becomes close to that of ionization, thus, establishing equilibrium. Therefore, in reality the ionization-layer edge is determined by recombination. A simplified way to attack the problem is to consider that in the steady or quasi-steady discharge, high plasma density and large electron temperature will lead to establishing a fully ionized plasma. Therefore, the ionization layer is the region where transition to fully ionized plasma occurs (Fig. 9). We are starting our consideration from the Knudsen-layer edge. The reason for this is that, under typical conditions, the mean free path is much larger than the Debye length, and therefore, sheath thickness is much smaller than that of the Knudsen-layer length. It is assumed that electron impact ionization is the dominant process in the ionization layer, whereas recombination is unimportant. ${ }^{35}$ In this section, we describe the asymptotic behavior of the solution of the ionization-layer problem in the case of strong acceleration, that is, strong effect of magnetic field in the ionization layer. This case corresponds to the micro-PPT conditions. We assume that the smooth sonic transition takes place in the ionization layer. Using L'Hopital's rule, one can find a velocity gradient near the sonic plane, which is finite under this condition. This procedure was used by a number of authors. ${ }^{36,37}$ 


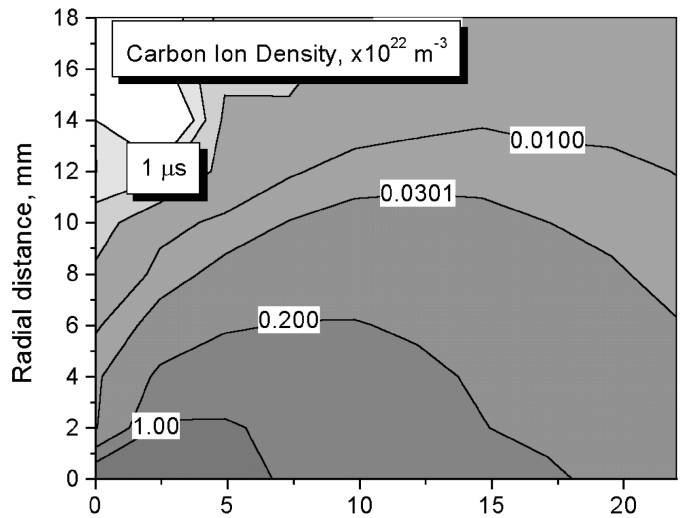

Axial distance, $\mathrm{mm}$

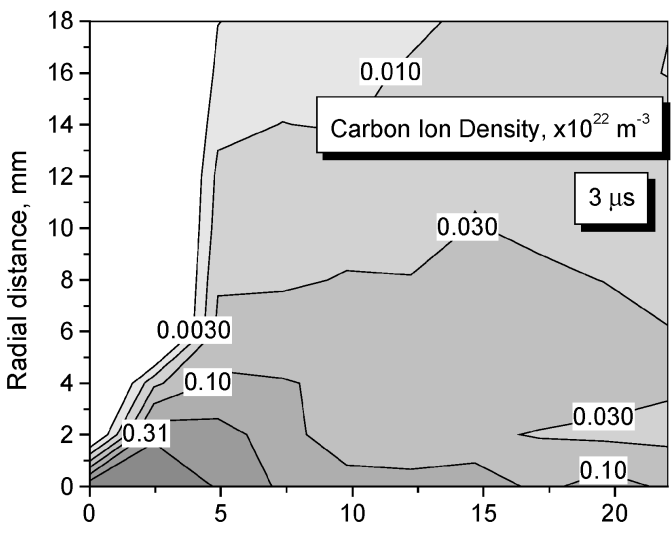

Axial distance, $\mathrm{mm}$
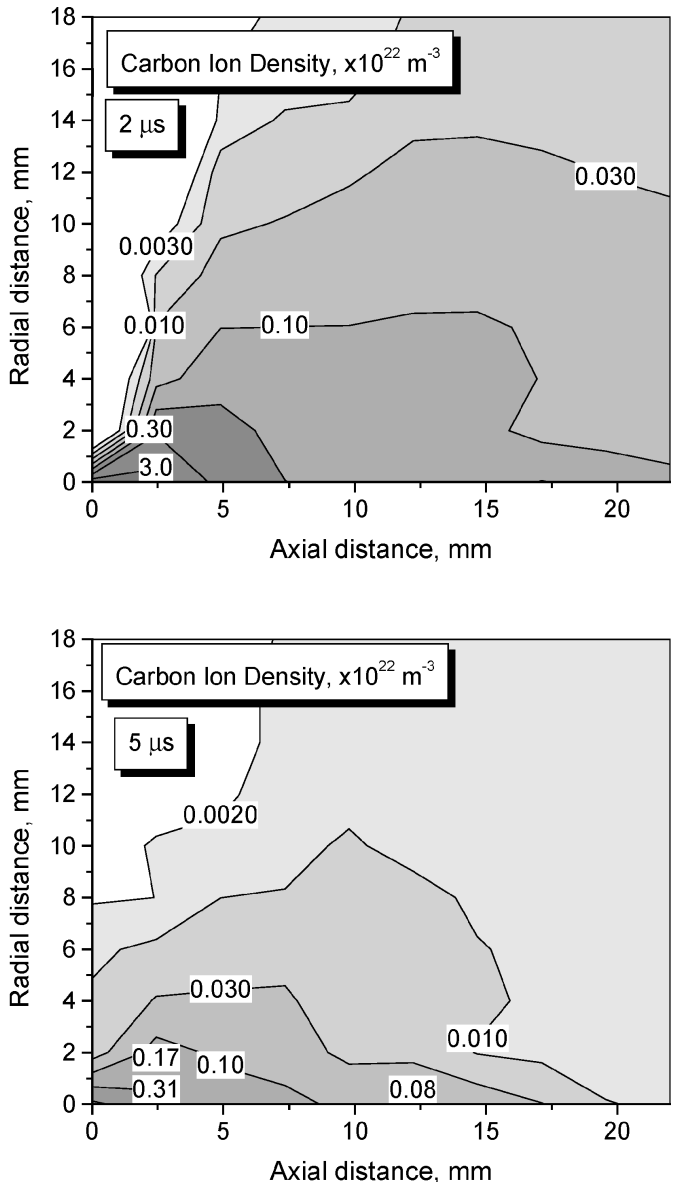

Axial distance, $\mathrm{mm}$

Fig. 5 Evolution of the carbon ion density during the pulse.
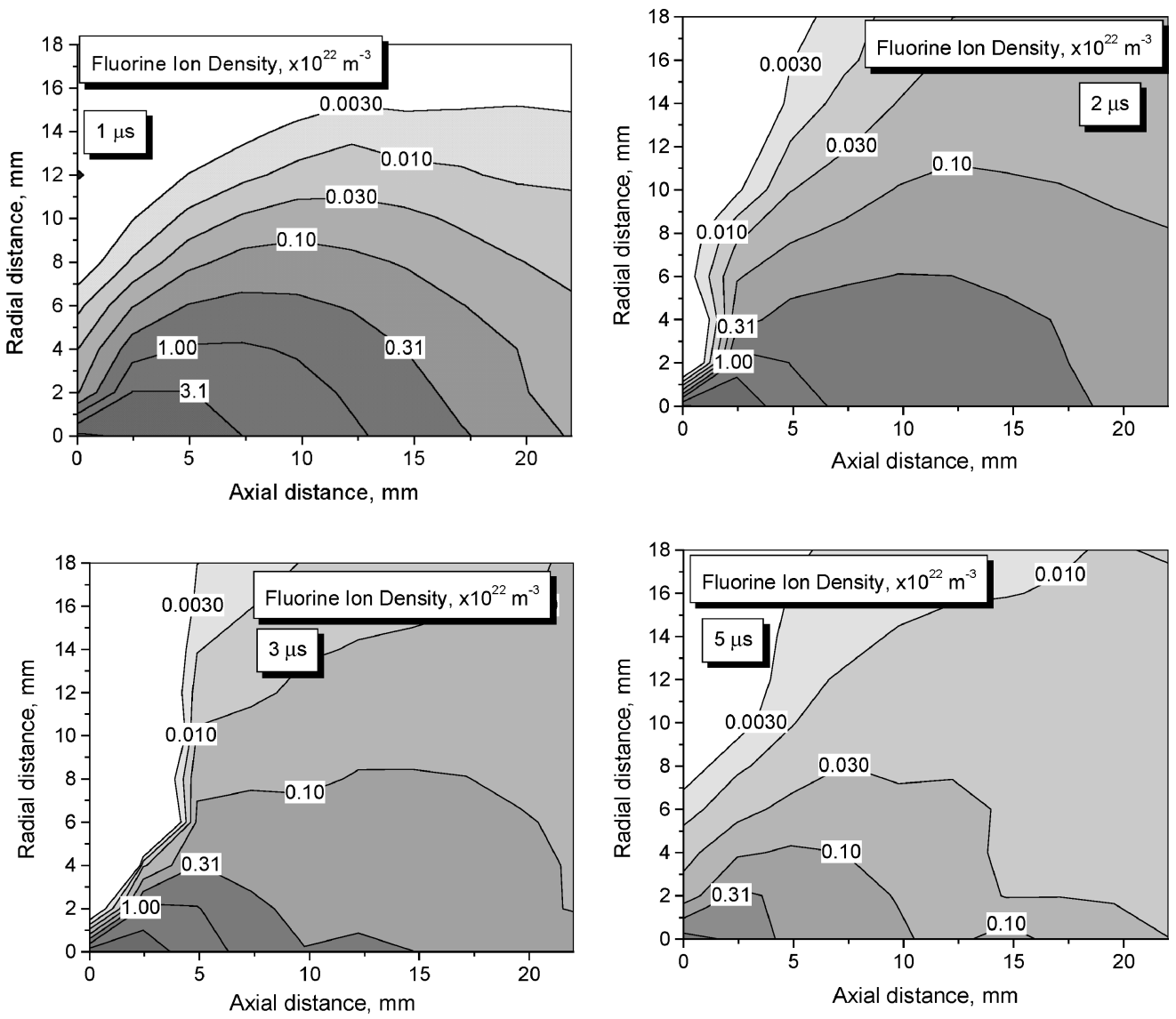

Fig. 6 Evolution of the fluorine ion density during the pulse. 

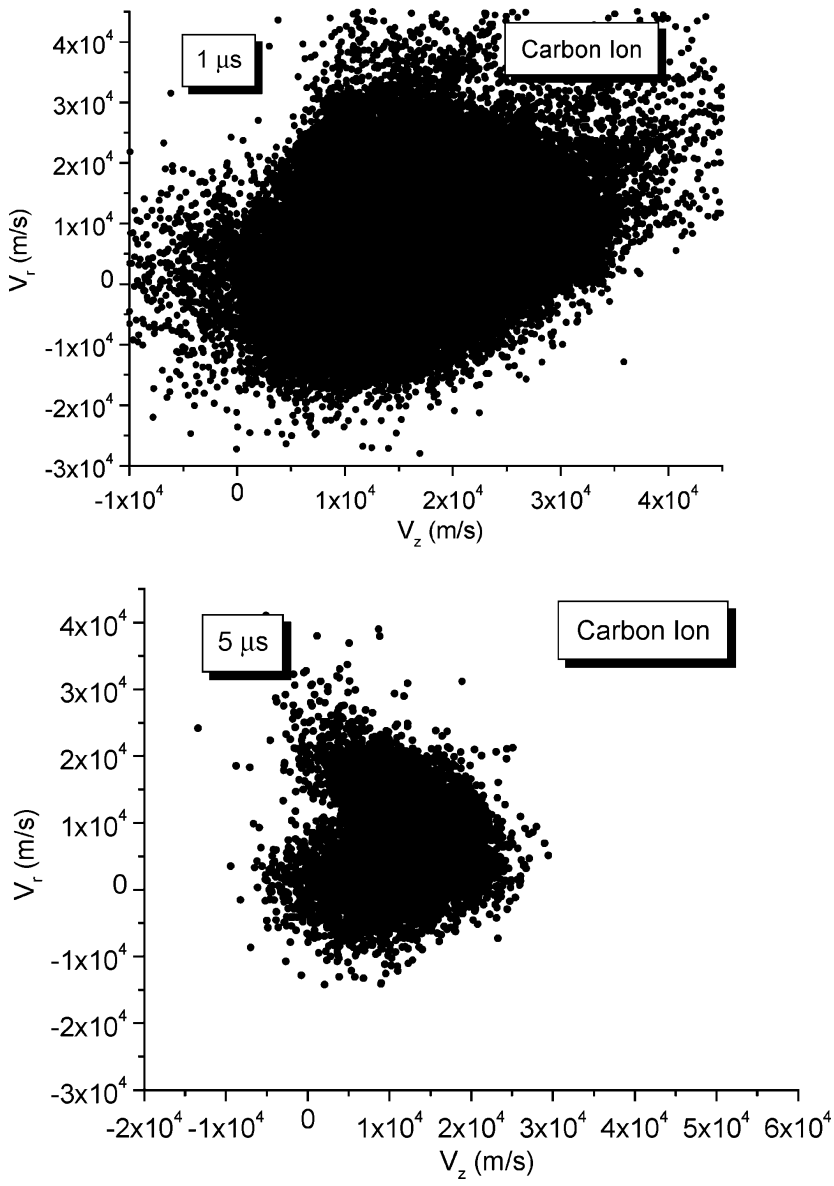

Fig. 7 Ion velocity phase (carbon).

The solution that allows smooth transition has the following expression for maximal electron density (normalized by the total particle density) in the vicinity of the sonic point ${ }^{35}$ :

$$
n=0.5\left[1-\sqrt{1-\left(\alpha \beta / \varepsilon^{0.5}\right)(1-1 / \beta)}\right]
$$

where $\alpha=C_{i a} /\left(r_{a} \alpha_{i} N_{1}\right), \beta=\left(V_{a} / C_{s}\right)^{2}, \varepsilon=\left(T_{i}+T_{e}\right) / T_{i}$, and $V_{a}$ is the Alfven wave speed,

$$
V_{a}=B_{1} /\left(\mu N_{1} m\right)^{0.5}
$$

where $C_{i a}$ is the heavy particle thermal velocity and $B_{1}$ is the magnetic field at the edge of the Knudsen layer. To calculate the ionization rate $\alpha_{i}$ for the $\mathrm{C}-\mathrm{F}$ plasma, the electron impact ionization cross sections (available from a database ${ }^{38}$ ) were used. Equation (9) is an expression for density behavior near the sonic plane in the case of the regular sonic transition. According to the nonequilibrium model [Eq. (9)], the degree of ionization is affected by an additional parameter, which is the ratio of the electron temperature at the beginning of ionization layer [in Eq. (9)] to the electron temperature in the plasma bulk [calculated from Eq. (1)]. The physical meaning of this parameter is that there is the electron temperature gradient from the plasma bulk to the propellant surface as shown in Fig. 9. In this model, the ratio of the electron temperature at the beginning of the ionization layer [used to calculate ionization degree according to Eq. (9)] to the bulk electron temperature $\theta$ is used as a parameter.

\section{B. Experimental Data and Comparison with Model Predictions}

An experimental basis for comparison is provided using a twocolor interferometer. Electron and neutral density measurements are taken on a $6.35-\mathrm{mm}-\left(\frac{1}{4}\right.$-in- $)$ diam microPPT at AFRL. The interferometer uses 488- and 1152-nm wavelengths and a quadrature heterodyne technique described by Spanjers et al. ${ }^{39}$ The two-color
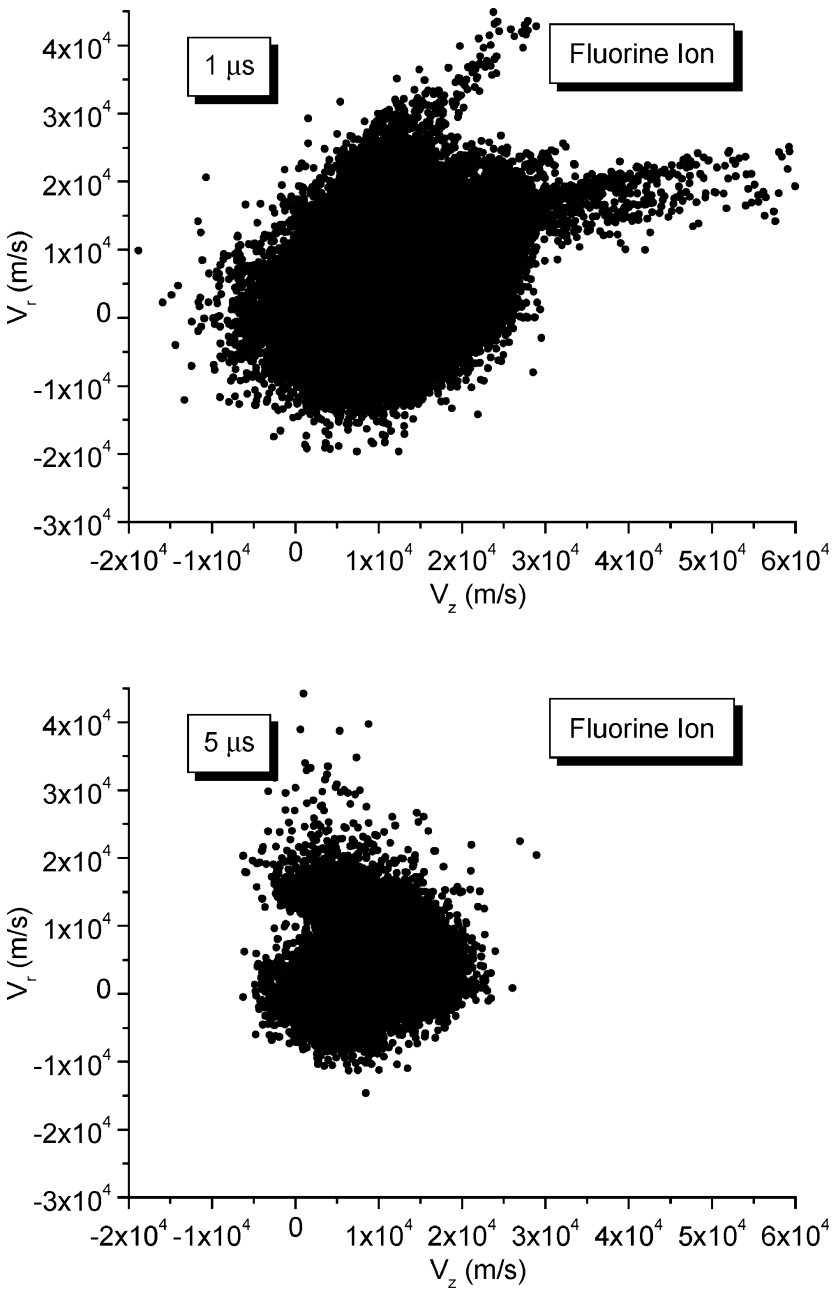

Fig. 8 Ion velocity phase (fluorine).

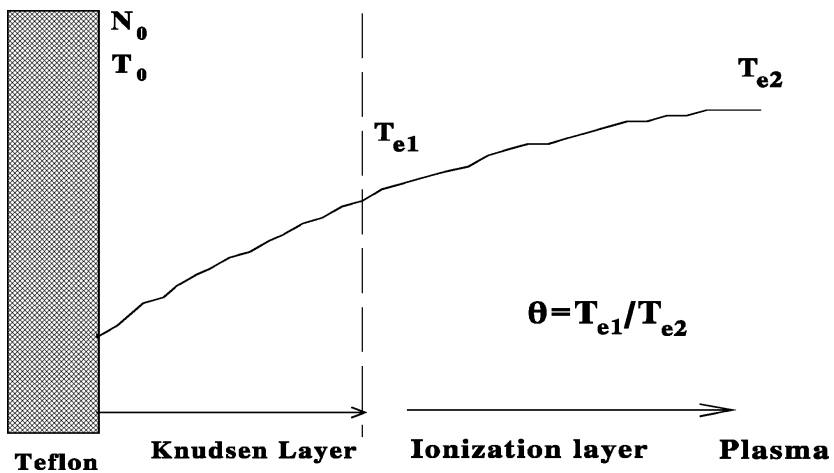

Fig. 9 Schematic of the ionization region near the Teflon surface.

technique uses the difference in phase shifts from the two wavelengths to calculate electron and neutral densities. In this case, the data are taken with scene and reference beam focused on the detector faces. This technique tends to ensure that both lasers are sampling the same portions of the plume. ${ }^{40}$ Older data with less focusing are shown with typical uncertainty by Antonsen et al..$^{41}$ along with a more thorough treatment of the two-color diagnostic. The data in Fig. 10 have a maximum uncertainty of $\pm 1.15 \times 10^{16} \mathrm{~cm}^{-3}$ for electron density and $\pm 1.76 \times 10^{16} \mathrm{~cm}^{-3}$ for neutral density at $2 \mu \mathrm{s}$ into the discharge. For the data shown here, the beam center is located $3 \mathrm{~mm}$ from fuel face on the thruster centerline with a beam diameter of $6 \mathrm{~mm}$. Note that the discharge in the micro-PPT may be azimuthally nonuniform (nonaxisymmetric). ${ }^{42}$ Strictly speaking, a comparison between an axisymmetric model and experiment in this 


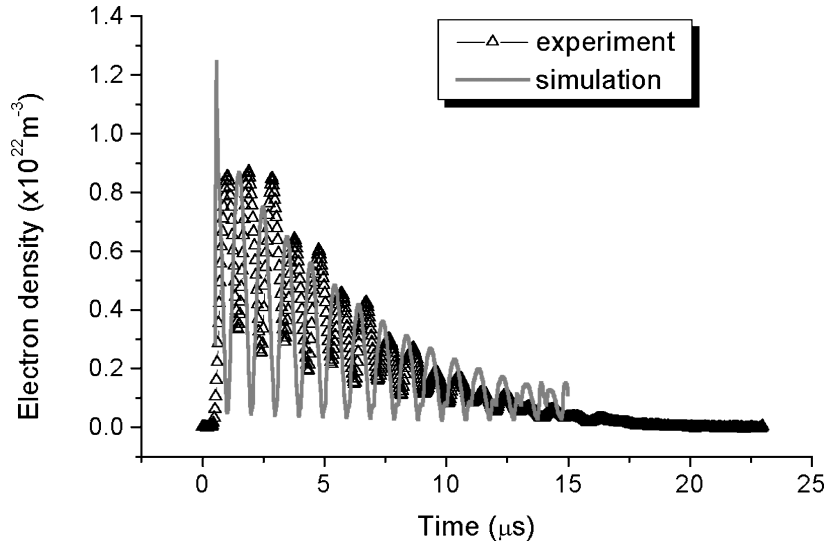

a)

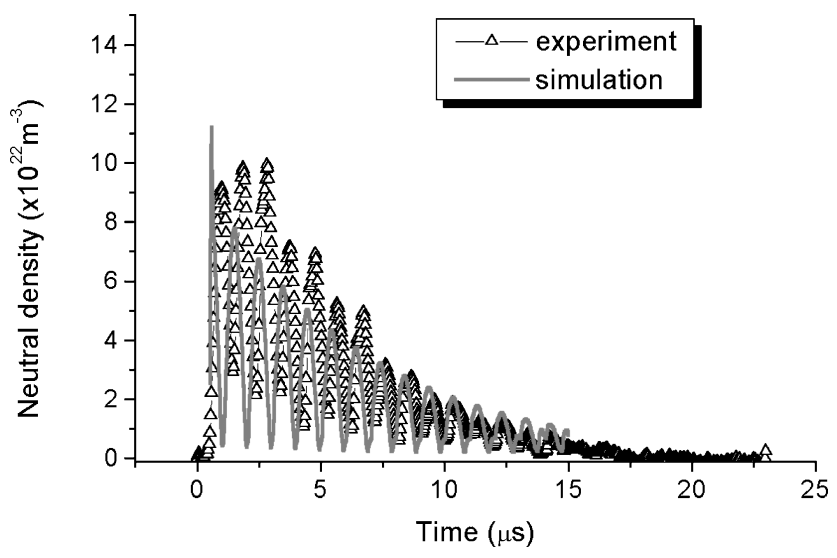

b)

Fig. 10 Comparison of predicted and measured electron and neutral densities time variation at $3 \mathrm{~mm}$ from the propellant face at the axis in the case of the $6.35-\mathrm{mm}$-diam micro-PPT firing at $6 \mathrm{~J}, \theta=0.2$ : a) plasma density and b) neutral density.

case may be questionable. However, the mentioned nonuniformity decreases with discharge energy, ${ }^{42}$ and experimental data (used for comparison in this paper) are reproducible during many pulses. This suggests that any effect of nonuniformity may be small in this case and our comparison is justified.

Figure 10 shows the experimental data along with model predictions. Plasma density peaks at about $1 \times 10^{22} \mathrm{~m}^{-3}$ and decreases by several orders of magnitude toward the pulse end. The neutral density is significantly higher and peaks at about $11 \times 10^{22} \mathrm{~m}^{-3}$. The experimental data were taken at a discharge energy of $6.0 \mathrm{~J}$ from a $0.417-\mu \mathrm{F}$ capacitor. A comparison of the simulation and experimental results is shown in Fig. 10 with $\theta$ as a parameter. One can see that the model predicts well both the plasma and neutral density level and temporal behavior during the entire pulse. Note that our estimations show that the electron temperature change across the ionization layer is close to that assumed in the model, that is, $\theta=0.2$, for typical micro-PPT operation parameters. In future work, we intend to determine this free parameter.

\section{Conclusions}

In this paper, a self-consistent description of an axisymmetric electromagnetic PPT from plasma generation into the near plume was presented. A micro-PPT developed at AFRL was considered as a working example. In this device, no separation exists between the main plasma acceleration region, which usually occurs in an internal flow, and the external plasma plume field. Therefore, a single endto-end model is necessary for accurate plume simulations. A kinetic Teflon ablation model was incorporated to provide the boundary conditions for the plasma plume simulation.

Phenomena in the plasma plume related to electromagnetic effects were studied. The plume simulation showed that a high-density re- gion is developed at a few millimeters from the thruster exit plane at the axis. This region exists during the entire pulse, but the plasma density decreases from about $2 \times 10^{22} \mathrm{~m}^{-3}$ at the beginning of the pulse down to $0.3 \times 10^{22} \mathrm{~m}^{-3}$ at $5 \mu \mathrm{s}$. The velocity phase is centered at about $20 \mathrm{~km} / \mathrm{s}$ in the axial direction, demonstrating that the micro-PPT is essentially an electromagnetic accelerator. At a later stage of the pulse, there are two ion populations with positive and negative radial velocity. It is predicted that there is a population of ions having a negative axial velocity with magnitude up to about $10 \mathrm{~km} / \mathrm{s}$. This population creates the backflow contamination that flows mainly onto the thruster itself. The carbon ions have a larger negative velocity due to their higher mobility that results in their domination in the backflux. It is believed that this backflux may be responsible for charring phenomena observed in this thruster. Predicted electron and neutral density in the near-field plume was compared directly with experimental data and good agreement was obtained.

\section{Acknowledgment}

The first two authors gratefully acknowledge financial support by the Air Force Office of Scientific Research through Grant F4962002-1-0084.

\section{References}

${ }^{1}$ Burton, R. L., and Turchi, P. J., "Pulsed Plasma Thruster," Journal of Propulsion and Power, Vol. 14, No. 5, 1998, pp. 716-735.

${ }^{2}$ Vondra, R. J., and Thomassen, K. I., "Flight Qualified Pulsed Plasma Thruster for Satellite Control," Journal of Spacecraft and Rockets, Vol. 11, No. 9, 1974, pp. 613-617.

${ }^{3}$ Turchi, P. J., "Directions for Improving PPT Performance," Proceeding of the 25th International Electric Propulsion Conference, Vol. 1, The Electric Propulsion Society, Worthington, OH, 1998, pp. 251-258.

${ }^{4}$ Choueiri, E. Y., "System Optimization of Ablative Pulsed Plasma Thruster for Stationkeeping," Journal of Spacecraft and Rockets, Vol. 33, No. 1, 1996, pp. 96-100.

${ }^{5}$ Spores, R. A., Cohen, R. B., and Birkan, M., "The USAF Electric Propulsion Program," Proceedings of the 25th International Electric Propulsion Conference, Vol. 1, The Electric Propulsion Society, Worthington, OH, 1997, p. 1 .

${ }^{6}$ Gatsonis, N. A., and Yin, X., "Axisymmetric DSMC/PIC Simulation of Quasi-neutral Partially Ionized Jets," AIAA Paper 97-2535, July 1997.

${ }^{7}$ Boyd, I. D., Keidar, M., and McKeon, W., "Modeling of a Pulsed Plasma Thruster from Plasma Generation to Plume Far Field," Journal of Spacecraft and Rockets, Vol. 37, No. 3, 2000, pp. 399-407

${ }^{8}$ Keidar, M., and Boyd, I. D., "Device and Plume Model of an Electrothermal Pulsed Plasma Thruster," AIAA Paper 2000-3430, July 2000.

${ }^{9}$ Hoskins, W. A., and Cassady, R. J., "Applications for Pulsed Plasma Thrusters and the Development of Small PPTs for Microspacecraft," AIAA Paper 2000-3434, July 2000.

${ }^{10}$ Dunning, J. W., Benson, S., and Oleson, S., "NASA's Electric Propulsion Program," 27th International Electric Propulsion Conf., Paper IEPC01-002, Oct. 2001.

${ }^{11}$ Zakrzwsky, C., Benson, S., Sanneman, P., and Hoskins, A., "On-Orbit Testing of the EO-1 Pulsed Plasma Thruster," AIAA Paper 2002-3973, July 2002.

${ }^{12}$ Gulczinski, F., III, Dulligan, M., Lakes, J., and Spanjers, G., "Micropropulsion Research at AFRL," AIAA Paper 2000-3255, July 2000.

${ }^{13}$ Mikellides, P. G., and Turchi, P. J., "Modeling of Late-Time Ablation in Teflon Pulsed Plasma Thruster," AIAA Paper 96-2733, July 1996.

${ }^{14}$ Keidar, M., Fan, J., Boyd, I. D., and Beilis, I. I., "Vaporization of Heated Materials into Discharge Plasmas," Journal of Applied Physics, Vol. 89, No. 6, 2001, pp. 3095-3098.

${ }^{15}$ Keidar, M., Boyd, I. D., and Beilis, I. I., "On the Model of Teflon Ablation in an Ablation-Controlled Discharge," Journal of Physics D: Applied Physics, Vol. 34, June 2001, pp. 1675-1677.

${ }^{16}$ Keidar, M., and Boyd, I. D., "Effect of a Magnetic Field on the Plasma Plume from Hall Thruster," Journal of Applied Physics, Vol. 86, No. 9, 1999, pp. 4786-4791.

${ }^{17}$ Keidar, M., Boyd, I. D., and Beilis, I. I., "Electrical Discharge in the Teflon Cavity of a Co-Axial Pulsed Plasma Thruster," IEEE Transactions on Plasma Science, Vol. 28, No. 2, 2000, pp. 376-385.

${ }^{18}$ Keidar, M., Boyd, I. D., and Beilis, I. I., "Model of an Elecrothermal Pulsed Plasma Thruster," Journal Propulsion and Power, Vol. 19, No. 3, 2003, pp. 424-430. 
${ }^{19}$ Zemskov, A. I., Prut, V. V., and Khrabrov, V. A., "Pulsed Discharge in Dielectric Chamber," Soviet Physics-Technical Physics, Vol. 17, 1972, pp. 285-289.

${ }^{20}$ Kozlov, G. I., Kuznetsov, V. A., and Masyukov, V. A., "Radiative Losses by Argon Plasma and the Emissive Model of a Continuos Optical Discharge," Soviet Physics-JETP, Vol. 39, No. 3, 1974, pp. 463-468.

${ }^{21}$ Raizer, Y. P., Gas Discharge Physics, Nauka, Moscow, 1987 (in Russian), p. 229.

${ }^{22}$ Kovitya, P., "Thermodynamic and Transport Properties of Ablated Vapors of PTFE, Alumina, Perspex and PVC in the Temperature Range 500030000 K," IEEE Transactions on Plasma Science, Vol. 12, No. 1, 1984, pp. $38-42$

${ }^{23}$ Schmahl, C. S., and Turchi, P. J., "Development of Equation-of-State and Transport Properties for Molecular Plasmas in Pulsed Plasma Thrusters. Part I: A Two-Temperature Equation of State for Teflon," Proceedings of the International Electric Propulsion Conference, 1997, pp. 781-788.

${ }^{24}$ Griem, H. R., Plasma Spectroscopy, McGraw-Hill, New York, 1964.

${ }^{25}$ Andrianov, A. M., Zemskov, A. I., Prut, V. V., and Khrabrov, V. A., "Pulsed Discharges in Dielectric Chambers," Soviet Physics-Technical Physics, Vol. 14, No. 2, 1969, pp. 318-321.

${ }^{26}$ Bird, G. A., Molecular Gas Dynamics and the Direct Simulation of Gas Flows, Clarendon, Oxford, 1994.

${ }^{27}$ Dalgarno, A., McDowell, M. R. C., and Williams, A., "The Modilities of Ions in Unlike Gases," Proceedings of the Royal Society of London, Vol. 250, April 1958, pp. 411-425.

${ }^{28}$ Sakabe, S., and Izawa, Y., "Simple Formula for the Cross Sections of Resonant Charge Transfer Between Atoms and their Ions at Low Impact Velocity," Physical Review A: General Physics, Vol. 45, No. 3, 1992, pp. 2086-2089.

${ }^{29}$ Birdsall, C. K., and Langdon, A. B., Plasma Physics via Computer Simulation, Adam Hilger, Bristol, England, U.K., 1991.

${ }^{30}$ Beilis, I. I., Keidar, M., and Goldsmith, S., "Plasma-Wall Transition: The Influence of the Electron to Ion Current Ratio on the Magnetic Presheath Structure," Physics of Plasmas, Vol. 4, No. 10, 1997, pp. 3461-3468.

${ }^{31}$ Popov, G. A., Kazeev, M. N., and Kozlov, V. F., "Two Dimensional Nu- merical Simulation of Co-Axial APPT," 27th International Electric Propulsion Conf., Paper IEPC 2001-159, Oct. 2001.

${ }^{32}$ Kingsep, A. S., Mokhov, Y. V., and Chukbar, K. V., "Nonlinear Skin Effect in Plasmas," Soviet Journal of Plasma Physics, Vol. 10, No. 4, 1984, pp. 495-498.

${ }^{33}$ Brushlinskii, K. V., and Morozov, A. I., "Calculation of TwoDimensional Plasma Flows in Channels," Rev. Plasma Physics, edited by M. A. Leontovich, Vol. 8, Consultants Bureau, New York, 1980.

${ }^{34}$ Keidar, M., Boyd, I. D., Gulczinski, F., III, Antonsen, E. L., and Spanjers, G. G., "Analyses of Teflon Surface Charring in a Micro-Pulsed Plasma Thruster," 27th International Electric Propulsion Conf., Paper IEPC-01-155, 2001.

${ }^{35}$ Keidar, M., and Boyd, I. D., "Ionization Non-Equilibrium and Ablation Phenomena in a Micro-Pulsed Plasma Thruster," AIAA Paper 2002-4275, July 2002.

${ }^{36}$ Fruchtman, A., Fisch, N. J., and Raitses, Y., "Control of the Electric Field Profile in the Hall Thruster," Physics of Plasmas, Vol. 8, No. 3, 2001, pp. 1048-1056.

${ }^{37}$ Ahedo, E., Martinez-Cerezo, P., and Martinez-Sanchez, M., "Onedimensional Model of the Plasma Flow in a Hall thruster," Physics of Plasmas, Vol. 8, No. 6, 2001, pp. 3058-3067.

${ }^{38}$ International Atomic Energy Agency (IAEA) Aladdin Database, URL: http://www-amdis.iaea.org/aladdin.html.

${ }^{39}$ Spanjers, G. G., McFall, K. A., Gulczinski, F., III, and Spores, R. A., "Investigation of Propellant Inefficiencies in a Pulsed Plasma Thruster," AIAA Paper 96-2723, July 1996.

${ }^{40}$ Antonsen, E. L., "Herriott Cell Interferometry for Pulsed Plasma Density Measurements," M. S. Thesis, Univ. of Illinois at Urbana-Champaign, IL, May 2001

${ }^{41}$ Antonsen, E. L., Burton, R., and Spanjers, G. G., "High Resolution Laser Diagnostics in Millimeter-Scale Micro Pulsed Plasma Thrusters," 27th International Electric Propulsion Conf., Paper IEPC 2001-157, Oct. 2001.

${ }^{42}$ Keidar, M., Boyd, I. D., Antonsen, E. L., and Spanjers, G. G., "Progress in Development of Modeling Capabilities for a Micro-Pulsed Plasma Thruster," AIAA Paper 2003-5166, July 2003.

\section{Economic Principles Applied to Space Industry Decisions Joel S. Greenberg, Princeton Synergetics, Inc.}

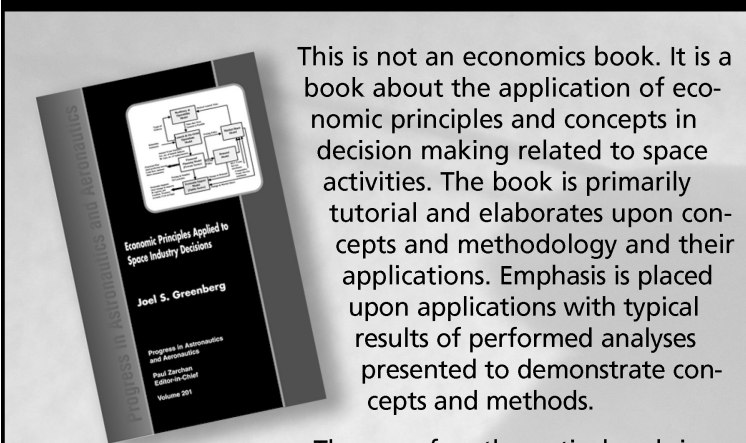

The use of mathematical and simulation models serves as the underpinning for much of the presented materials. The specific models considered have been selected to demonstrate the role that a structured thought process can play in the decision process. Since most decisions relating to technology development, product design, capital expenditures, and investments involve uncertainty and risk, a number of the selected models, developed methodologies, and presented examples explicitly and quantitatively consider uncertainty and risk.
The objective of this book is to put economic analysis into perspective with respect to real-world decision making in the space industry. It will expand the perspective of the reader with respect to the type of tools and analyses that might be brought to bear on complex business and government problems.

\section{Contents:}

Introduction • Investment Decisions • RLV Economics - Space Operations $\bullet$ Licensing and Regulatory Issues - Beyond Space: Energy and Gaming - Appendix: Estimating the Likelihood of Investment

Progress in Astronautics and Aeronautics Series 2003, 480 pages, Hardback

ISBN: 1-56347-607-X

List Price: $\$ 100.95$

AIAA Member Price: $\$ 69.95$

Publications Customer Service, P.O. Box 960

Herndon, VA 20172-0960

Phone: 800/682-2422; 703/661-1595

Fax: 703/661-1501

E-mail:warehouse@aiaa.org•Web: www.aiaa.org 